\title{
Neurotoxicity of subarachnoid Gd-based contrast agent accumulation: a potential complication of intraoperative MRI?
}

\author{
*Monika Lauer, MD, ${ }^{1}$ Arne Lauer, MD, ${ }^{1}$ Se-Jong You, MD,, Sara Kluge, MD, ${ }^{1}$ Elke Hattingen, MD, \\ Patrick N. Harter, MD, ${ }^{2,3}$ Christian Senft, MD, ${ }^{4}$ Marlies Wagner, MD, ${ }^{1}$ and Martin Voss, MD ${ }^{5}$ \\ ${ }^{1}$ Institute of Neuroradiology, University Hospital Frankfurt, Goethe University, Frankfurt am Main; ${ }^{2}$ Edinger Institute (Institute of \\ Neurology), Goethe University, Frankfurt am Main; ${ }^{3}$ German Cancer Research Center (DKFZ) Heidelberg, and German Cancer \\ Consortium (DKTK) Frankfurt/Mainz; ${ }^{4}$ Department of Neurosurgery, University Hospital Frankfurt, Goethe University, Frankfurt \\ am Main; and ${ }^{5}$ Dr. Senckenberg Institute of Neurooncology, University Hospital Frankfurt, Goethe University, Frankfurt am Main, \\ Germany
}

OBJECTIVE Intraoperative MRI with Gd-based contrast agent (GBCA) improves the extent of resection of contrast-enhancing brain tumors. Signal changes of CSF due to perioperative GBCA leakage in the subarachnoid space have been reported. However, although GBCA potentially exhibits neurotoxic effects, so far no associated complications have been described. In this case series, the authors report a single-center cohort of patients with subarachnoid GBCA extravasation after intraoperative MRI and discuss potential neurotoxic complications and potential ways of avoiding them.

METHODS All patients with CSF signal increase on unenhanced T1-weighted and FLAIR images on postoperative MRI, who had previously undergone tumor resection with use of intraoperative MRI, were retrospectively included and compared with a control cohort. The control group was matched in age, tumor characteristics, and extent of resection; comparisons were made regarding postoperative seizures and ICU stay. A subgroup with initially diagnosed malignant glioma was additionally analyzed for potential delay of initiation of adjuvant treatment and overall survival.

RESULTS Seven patients with postoperative GBCA accumulation in the subarachnoid space were identified; 5 presented with focal seizures and altered mental status postoperatively. Poor patient condition led to extended ICU stay and prolonged delay of the initiation of adjuvant treatment in patients with newly diagnosed malignant glioma. Overall survival was reduced compared to the matched control group.

CONCLUSIONS The results suggest that there might be a risk of neurotoxic complications if GBCA that is intravenously applied during neurosurgery leaks into the subarachnoid space. Patients with highly vascularized tumors with intraoperative bleeding seem to be especially at risk for GBCA accumulation and neurotoxic complications. Therefore, awareness of the potential risk of complicating GBCA leakage is mandatory in the application of intraoperative GBCA.

https://thejns.org/doi/abs/10.3171/2020.10.FOCUS20402

KEYWORDS intraoperative MRI; Gd; toxicity; glioblastoma; gadolinium encephalopathy

$\mathrm{I}$ $\mathrm{N}$ malignant brain tumors gross-total resection is associated with increased progression-free survival (PFS) and overall survival (OS).,2 Usually MRI examinations with intravenous application of Gd-based contrast agent (GBCA) are performed before and after surgery to evaluate the extent of resection. Consequently, contrast-enhancing residual tumor is detected first in the postoperative MRI examination. Intraoperative MRI examinations, however, are obtained during surgery, and possible tumor remnants are detected as enhancing regions after intravenous application of a GBCA, allowing immediate further tumor resection. Thus, intraoperative MRI is a method proven to improve the extent of resection of contrast-enhancing malignant brain tumors, which is associated with increased PFS and OS. ${ }^{1,2}$

Usually two additional contrast-enhanced scans are performed during tumor neurosurgery-one prior to the tumor resection to provide neuronavigation and one after the resection to assess the extent of resection. GBCA leakage in the subarachnoid space has been reported after intravenous injection in patients with an impaired blood-

ABBREVIATIONS BBB = blood-brain barrier; GBCA = Gd-based contrast agent; KPS = Karnofsky Performance Scale; MGMT = 06-methylguanine DNA methyltransferase; $O S=$ overall survival; $P F S=$ progression-free survival.

SUBMITTED May 14, 2020. ACCEPTED October 8, 2020.

INCLUDE WHEN CITING DOI: $10.3171 / 2020.10$.FOCUS20402.

${ }^{*}$ M.W. and M.V. contributed equally to this work. 
brain barrier (BBB) - for example, due to surgery. ${ }^{3,4}$ High doses of GBCA in the subarachnoid space are known to potentially result in neurotoxic manifestations, which may include seizures and an altered mental status - a condition that has been called "gadolinium encephalopathy." ever, neurotoxic complications following intraoperative MRI with intravenous GBCA application have not yet been described.

In this series we report on 7 patients with MRI-proven subarachnoid GBCA accumulation and potential neurotoxic complications after brain tumor surgery with intraoperative MRI, and we discuss possible associations between subarachnoid GBCA accumulation and clinical symptoms.

\section{Methods \\ Study Population}

This retrospective study was approved by the institutional review board at our university. In our single-center cohort, postoperative MRI scans of patients with proven malignant glioma (all WHO grade IV), who underwent tumor resection between 2014 and 2016, were retrospectively screened for visible signs of subarachnoid GBCA accumulation in the postoperative high-field MRI (hyperintense signal of CSF in FLAIR and T1-weighted images). All available demographic and clinical data were retrospectively collected.

A control cohort of patients undergoing brain tumor surgery between 2014 and 2016 without signs of GBCA in the subarachnoid space on postoperative MRI, with or without intraoperative GBCA application, was generated from our institutional brain tumor database. We selected the best matches in terms of age, tumor entity, preoperative Karnofsky Performance Scale (KPS) scores, isocitrate dehydrogenase mutation status, $O^{6}$-methylguanine DNA methyltransferase (MGMT) promoter methylation, and extent of resection. Patients and the control group were analyzed and compared for necessity of postoperative ICU stay and incidence of systemic or focal epileptic seizures as a potential symptom of neurotoxicity.

In addition, a subgroup of previously untreated patients with initial diagnosis of malignant glioma was generated from the patient and control groups. These groups were compared with regard to the interval between surgery and the beginning of adjuvant radio- and chemotherapy. Delayed initiation of adjuvant tumor therapy was defined as therapy starting later than 3 months postoperatively. ${ }^{8,9}$ To evaluate OS, the date of the patient's death was recorded; if unknown, the date of last contact after initiation of best supportive care was documented instead.

\section{MR Imaging}

Intraoperative MRI scans for microneurosurgical resection were performed using a mobile intraoperative ultra-low-field MRI system (PoleStar N-20; Odin Medical Technologies and Medtronic) ${ }^{1}$ after GBCA injection (Gadovist $1.0 \mathrm{mmol} / \mathrm{ml}$ ) usually at a dose of $0.1 \mathrm{ml} / \mathrm{kg}$. In some cases higher doses with at least $0.2 \mathrm{ml} / \mathrm{kg}$ to increase contrast were reported. However, no records of the actual amount of injected GBCA were available.
Two scans were obtained during the resection-one before starting surgery to provide neuronavigation, and one intraoperative postresection scan to assess the extent of resection. All patients underwent high-field MRI at $3 \mathrm{~T}$ (Verio; Siemens) between 12 and 72 hours after surgery. The postoperative MRI was performed using a standard protocol including axial T2-weighted turbo spin echo sequence (TR $5170 \mathrm{msec}$, TE $92 \mathrm{msec}$ ); axial FLAIR sequence (TR $6630 \mathrm{msec}$, TE $131 \mathrm{msec}$, TI $2140 \mathrm{msec}$ ); diffusion-weighted imaging/apparent diffusion coefficient (TR $7600 \mathrm{msec}$, TE $88 \mathrm{msec}$ ); and T2*-weighted images (TR $631 \mathrm{msec}$, TE $19.9 \mathrm{msec}$ ). Axial T1-weighted spin echo sequences (TR $590 \mathrm{msec}$, TE $12 \mathrm{msec}$ ) were performed before and after intravenous application of a standard dose of GBCA (Gadovist $1.0 \mathrm{mmol} / \mathrm{ml} ; 0.1 \mathrm{ml} / \mathrm{kg}$ ). Furthermore, after GBCA application, a coronal contrast-enhanced T1-weighted gradient echo sequence (TR $261 \mathrm{msec}$, TE $4.12 \mathrm{msec}$ ) and a 3D T1-weighted volumetric interpolated breath-hold examination (VIBE) sequence (TR $4.9 \mathrm{msec}$, TE $1.83 \mathrm{msec}$ ) in sagittal orientation were acquired.

\section{Imaging Evaluation}

Based on the consensus of two experienced neuroradiologists, the signal intensity of the CSF on unenhanced T1-weighted images and/or FLAIR images of the postoperative high-field MRI was analyzed visually and categorized. In case of a clearly homogeneously increased signal intensity of the CSF, subarachnoid GBCA accumulation was suspected (Fig. 1).

\section{Results}

\section{Patient Population}

Seven patients with suspected GBCA accumulation were identified based on the hyperintense signal of the CSF in the subarachnoid and intraventricular space on T1weighted and FLAIR sequences of the postoperative highfield MRI (see Fig. 1). All of these individuals underwent intraoperative MRI with intravenous GBCA after primary tumor resection (see below). Three patients were female and 4 were male. The mean age was $61.1 \pm 11$ years (range 29-78 years). The median preoperative KPS score was 70. Histopathologically, 6 patients were diagnosed with glioblastoma and 1 patient with its morphological variant gliosarcoma (all WHO grade IV). Four patients with initial diagnosis of malignant glioma (3 glioblastoma, 1 gliosarcoma) underwent first-time tumor resection with intraoperative MRI. In the remaining 3 patients intraoperative MRI was used for resection of recurrent glioma (all glioblastoma). For further tumor characteristics see Table 1 .

The generated matching cohort consisted of 19 patients; 6 of these patients underwent intraoperative MRI. All patients had newly diagnosed glioblastoma (WHO grade IV). The mean age was $71 \pm 3.9$ years (range $65-81$ years). The median preoperative KPS score was 80 . None of these patients showed a signal increase but had a normal signal of the CSF after surgery.

\section{Surgery}

All patients underwent diagnostic MRI with GBCA prior to and after neurosurgical tumor resection, at least 12 

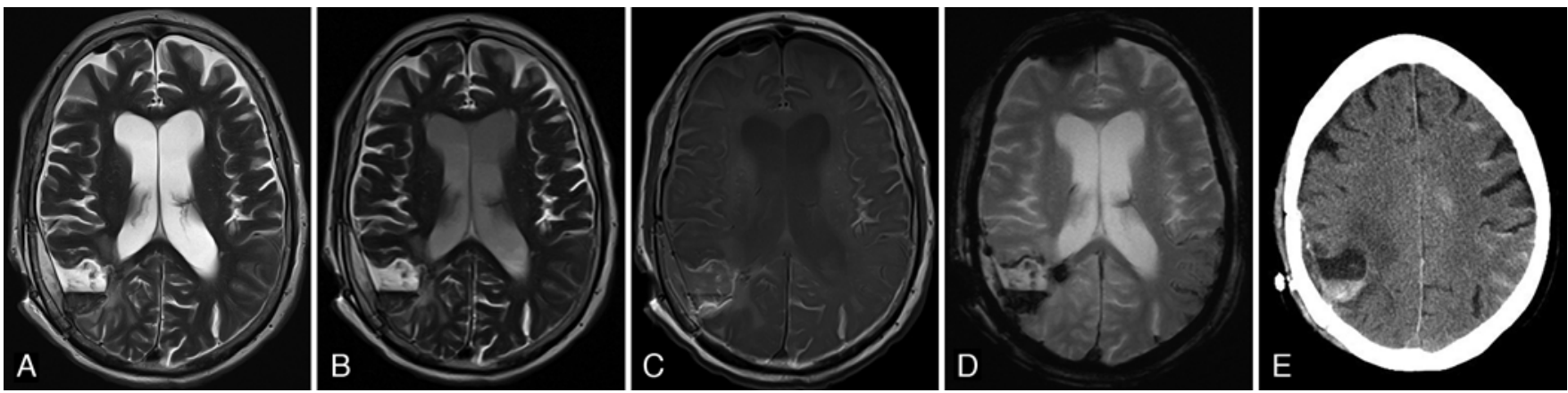

FIG. 1. Postoperative high-field MR images obtained in a patient after intraoperative MRI-guided neurosurgery of a glioblastoma in the right parietal lobe. Excessive intraoperative bleeding was reported in this patient. CSF signal appears normal in T2-weighted images (A), whereas FLAIR and T1-weighted images (B and C, respectively) revealed hyperintense signal changes of CSF due to the T1 shortening effect of GBCA. In T2* images (D) only a few sulci in the left parietal lobe show a signal loss as a sign of subarachnoid hemorrhage; however, extensive signal alterations corresponding to FLAIR and T1-weighted images can be excluded. Accordingly, CT images (E) display only a few hyperdense sulci as a correlate of subarachnoid blood. Excessive intraoperative bleeding explains the subarachnoid blood in distant contralateral sulci.

hours before and 12-72 hours after surgery. All patients undergoing intraoperative MRI received two additional scans with GBCA in the operative setting, one scan immediately before surgery and one during surgery to assess the extent of resection.

In 1 of 7 members of the patient group residual contrast-enhancing tissue was detected in the intraoperative MRI, and tumor resection was continued subsequently. In 5 of 7 patients with subarachnoid GBCA accumulation, excessive intraoperative bleeding due to high tumor vascularization was reported, resulting in extensive and timeconsuming hemostasis to control bleeding (see Table 2).

In the matched control group 4 patients with excessive intraoperative bleeding were reported; however, these patients did not receive intraoperative MRI, nor did they show homogeneous signal changes of the CSF on postoperative MRI. In contrast to the patient group, there was no further resection or excessive intraoperative bleeding in patients in the control group with intraoperative MRI (see Table 3).

\section{Clinical Course}

Four of the 7 patients with subarachnoid GBCA accumulation after neurosurgery presented with delayed or inadequate awakening (see Table 2). Later, 5 of the 7 patients had focal seizures during the postoperative ICU stay; of those, 2 patients developed a status epilepticus. These 5 patients remained in the ICU between 6 and 39 days (mean stay duration of 19.6 days). In only 2 of the 7 patients with a CSF signal increase in the postoperative FLAIR images was the clinical course unremarkable. These patients left the ICU 1 day after tumor resection (see Fig. 2).

In the matched control group, postoperative awakening and ICU stay were unremarkable in all patients, whether or not intraoperative MRI was performed (see Table 3). Matched control patients with intraoperative MRI left the ICU 1 day after surgery; patients without intraoperative MRI stayed 1.3 days (range 1-3 days).

\section{Adjuvant Treatment and OS}

In the group of patients with postoperative CSF GBCA accumulation, 4 of 7 patients were first diagnosed with malignant glioma (3 glioblastoma, 1 gliosarcoma). In 2 of these 4 patients the tumor had a methylated MGMT promoter, and in 2 patients the promoter methylation status was inconclusive. The median age at diagnosis was 73 years.

In the matched control group, the tumor of 9 patients had a methylated MGMT promoter, in 1 patient the methylation status was inconclusive, and in 9 patients the MGMT promoter was unmethylated. The median age in this group was 70 years.

TABLE 1. Demographic and tumor characteristics of 7 patients with postoperative GBCA accumulation

\begin{tabular}{|c|c|c|c|c|c|c|c|c|}
\hline \multirow{2}{*}{$\begin{array}{c}\text { Case } \\
\text { No. }\end{array}$} & \multicolumn{2}{|c|}{ Patient Characteristics } & \multicolumn{6}{|c|}{ Tumor Characteristics } \\
\hline & Age (yrs) & Sex & Tumor Entity & WHO Grade & IDHR132H & MGMT Promoter & Nuclear ATRX Expression & 1p/19q Deletion \\
\hline 1 & 73 & $\mathrm{~F}$ & Glioblastoma & IV & NA & Methylated & NA & NA \\
\hline 2 & 71 & $\mathrm{~F}$ & Glioblastoma & IV & No & Methylated & NA & No \\
\hline 3 & 78 & M & Gliosarcoma & IV & No & Inconclusive & NA & NA \\
\hline 4 & 73 & $\mathrm{~F}$ & Glioblastoma & IV & No & Methylated & Positive & NA \\
\hline 5 & 29 & M & Glioblastoma & IV & Yes & Methylated & Positive & Yes \\
\hline 6 & 52 & M & Glioblastoma & IV & No & Methylated & Positive & NA \\
\hline 7 & 52 & M & Glioblastoma & IV & No & Inconclusive & Positive & NA \\
\hline
\end{tabular}

$\mathrm{NA}=$ not applicable. 
TABLE 2. Surgery, postoperative care, and adjuvant treatment of patients with postoperative GBCA accumulation

\begin{tabular}{|c|c|c|c|c|c|c|c|c|c|}
\hline \multirow[b]{2}{*}{$\begin{array}{l}\text { Case } \\
\text { No. }\end{array}$} & \multicolumn{3}{|c|}{ Surgery } & \multicolumn{4}{|c|}{ Postop Care } & \multicolumn{2}{|c|}{ Adjuvant Therapy } \\
\hline & EOR & $\begin{array}{c}\text { Difficult } \\
\text { Hemostasis }\end{array}$ & $\begin{array}{l}\text { Continued } \\
\text { Resection }\end{array}$ & $\begin{array}{c}\text { Status } \\
\text { Epilepticus }\end{array}$ & $\begin{array}{c}\text { Focal } \\
\text { Seizures }\end{array}$ & $\begin{array}{c}\text { Delayed } \\
\text { Awakening }\end{array}$ & $\begin{array}{l}\text { ICU Stay } \\
\text { (days) }\end{array}$ & $\begin{array}{c}\text { Start After } \\
\text { Surgery (mos) }\end{array}$ & OS (mos) \\
\hline 1 & NTR & Yes & No & No & Yes & No & 21 & 1 & 8 \\
\hline 2 & GTR & Yes & No & Yes & Yes & Yes & 39 & No therapy & 21 \\
\hline 3 & GTR & Yes & No & No & Yes & Yes & 23 & No therapy & 5 \\
\hline 4 & NTR & Yes & No & Yes & Yes & Yes & 9 & No therapy & 5 \\
\hline 5 & GTR & No & No & No & No & No & 1 & 1 & \\
\hline 6 & GTR & No & No & No & Yes & Yes & 6 & 3 & 39 \\
\hline 7 & GTR & Yes & Yes & No & No & No & 1 & 0 & 14 \\
\hline
\end{tabular}

EOR = extent of resection; GTR = gross-total resection; NTR = near-total resection.

Cases 1, 3, 4, and 7 were newly diagnosed patients.

In the group with subarachnoid GBCA accumulation delayed initiation of adjuvant therapy ( $\geq 3$ months) was observed in 2 of the 4 newly diagnosed patients; in fact, they received no adjuvant treatment at all due to poor overall condition. In the other 2 patients, adjuvant therapy was not delayed. In the matched control group (with and without intraoperative MRI) adjuvant therapy was delayed in $1 \mathrm{pa}-$ tient (see Table 3).

The median OS was 6.5 months (range 5-14 months) for patients with postoperative GBCA accumulation, compared to 13 months for the matched control group (13 months [range 0-22 months] in the group without intraoperative MRI, and 13.5 months [range 4-37 months] in the group with intraoperative MRI).

\section{Discussion}

GBCA is in wide clinical use. The intravenous administration of GBCA is relatively safe and rarely causes systemic toxicity. "Off-label" reports also describe intrathecal administration-e.g., for myelography and cisternography, needle guidance during epidural spine procedures, and localization of spinal CSF leaks - in which low doses of GBCA have also been safely used with minor adverse effects (e.g., headache). ${ }^{10,11}$ However, an overdose intrathecal injection of GBCA can result in neurotoxic manifestations, which might be the reason for presumable clinical effects of the subarachnoid GBCA accumulation. The neurotoxic effects of GBCA are not completely understood. However, the toxicity of $\mathrm{Gd}$ is associated with similarity between $\mathrm{Gd}$ and $\mathrm{Ca}$ ions. Thus, $\mathrm{Gd}^{3+}$ can compete with $\mathrm{Ca}^{2+}$ and act as an inorganic blocker of voltage-gated Ca channels with negative consequences in many sites of the human body. ${ }^{12}$ Additionally, the neurotoxic effects seem to be proportional to the dose given and the contact time with the brain. ${ }^{3}$

Intraoperative MRI is applied in neurosurgery to detect residual tumor as an enhancing area after intravenous application of GBCA, allowing immediate further tumor resection. ${ }^{1}$ Consequently, it minimizes tumor remnants left unresected, which is associated with extended $\mathrm{OS},{ }^{13}$ and severe complications of intraoperative GBCA application have not been described so far.

Few studies describe a signal increase of the CSF on FLAIR images in early postsurgical MRI examinations after intraoperative MRI with intravenous injection of GBCA. Those signal changes may be the result of Gd leakage due to BBB breakdown from surgical manipulation in highly vascularized tumors. ${ }^{4}$ Free water such as CSF usually appears hypointense on T1-weighted and FLAIR images. If GBCA leaks into subarachnoid space, the T1-shortening effect of Gd results in a hyperintense signal of CSF on T1-weighted and FLAIR images. ${ }^{14}$

So far, no clinically noticeable adverse effects associated with the postsurgically increased signal of the CSF have been reported, although studies have shown detectable brain bio-electric changes after intravenous administration of paramagnetic contrast medium, especially in patients with CNS lesions causing BBB impairment. ${ }^{3}$ However, a systematic analysis of possible clinical effects associated with GBCA leakage has not been performed so far.

In our small single-center cohort, we found that most

TABLE 3. Overview of surgery, postoperative care for the whole cohort, and adjuvant treatment for newly diagnosed patients

\begin{tabular}{|c|c|c|c|c|c|c|c|c|c|}
\hline \multirow[b]{2}{*}{ Patient Group } & \multirow[b]{2}{*}{$\begin{array}{c}\text { No. of } \\
\text { Patients }\end{array}$} & \multicolumn{2}{|c|}{ Surgery } & \multicolumn{4}{|c|}{ Postop Care } & \multicolumn{2}{|c|}{ Adjuvant Therapy } \\
\hline & & $\begin{array}{c}\text { Difficult } \\
\text { Hemostasis }\end{array}$ & $\begin{array}{l}\text { Continued } \\
\text { Resection }\end{array}$ & $\begin{array}{c}\text { Status } \\
\text { Epilepticus }\end{array}$ & $\begin{array}{c}\text { Focal } \\
\text { Seizures }\end{array}$ & $\begin{array}{c}\text { Delayed } \\
\text { Awakening }\end{array}$ & $\begin{array}{l}\text { Mean ICU } \\
\text { Stay (days) }\end{array}$ & Delay & $\begin{array}{l}\text { Median OS } \\
\quad(\mathrm{mos})\end{array}$ \\
\hline No iMRI & 13 & 4 & NA & 0 & 0 & 0 & 1.25 & 1 & 13 \\
\hline iMRI, no postop Gadovist & 6 & 0 & 0 & 0 & 0 & 0 & 1.00 & 0 & 13.5 \\
\hline iMRI, postop Gadovist & 7 & 5 & 1 & 2 & 5 & 4 & $14.3 \pm 14$ & $2 / 4$ & 6.5 \\
\hline
\end{tabular}

iMRI = intraoperative MRI.

Values represent number of patients unless indicated otherwise. 


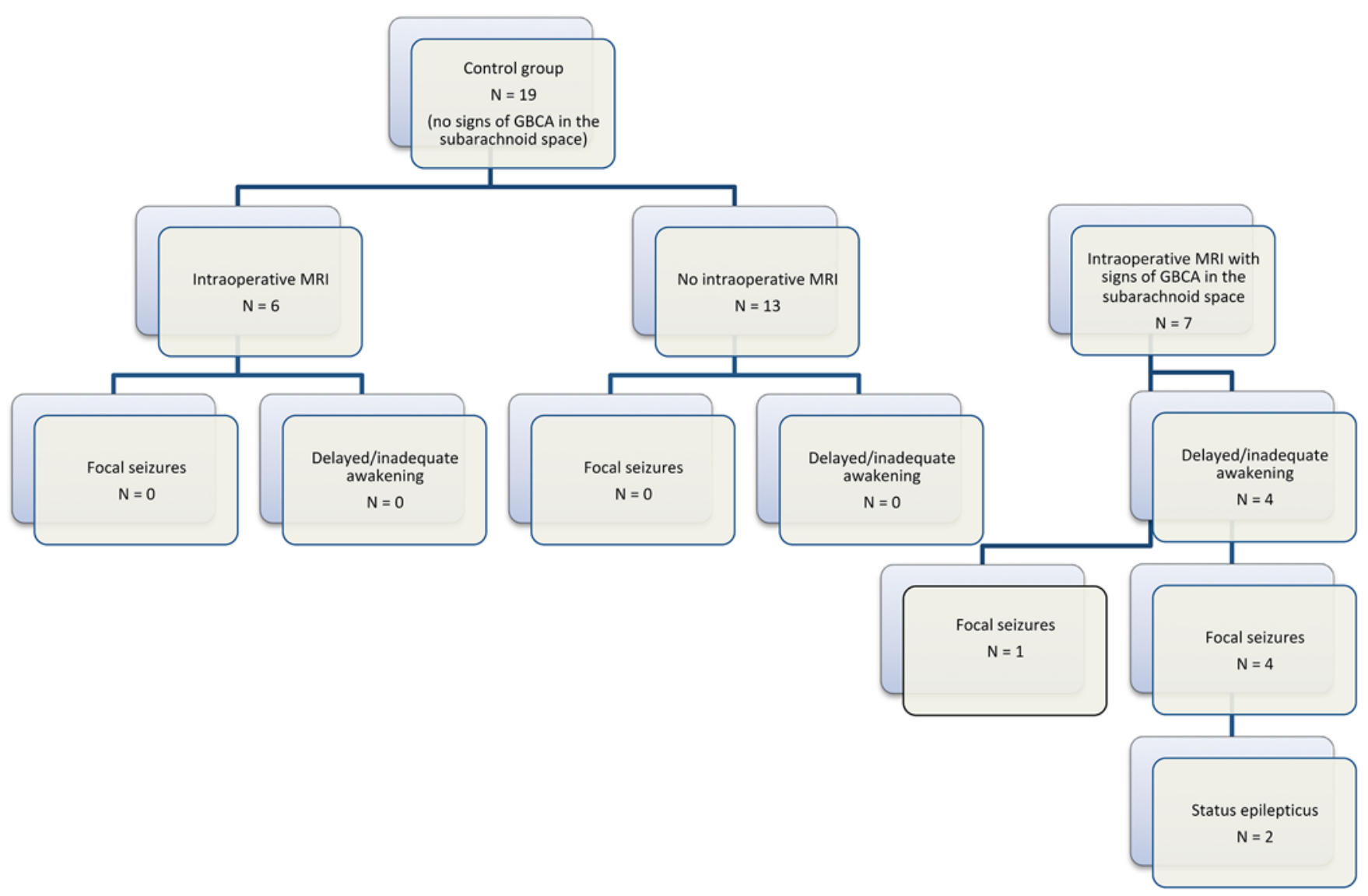

ICU $1 \mathrm{~d}$

FIG. 2. Flowchart of postoperative complications.

patients with visible subarachnoid GBCA accumulation required intensive care for a considerably longer period, and/or they had considerably more frequent focal seizures or a status epilepticus, compared to patients who had an intraoperative MRI sequence with GBCA but no subarachnoid contrast accumulation, or those who did not have intraoperative MRI.

Furthermore, initiation of adjuvant treatment was delayed in the group of patients with subarachnoid GBCA accumulation. Consequently, the median OS was considerably shorter (6.5 months) than in the control group, even though all patients had a complete tumor resection and mostly favorable molecular markers. In the control group patients with intraoperative MRI the median survival was 13.5 months, and in those without intraoperative MRI it was 13 months.

All patients who underwent an intraoperative MRI received an intravenous application of $0.1 \mathrm{mmol}$ GBCA at the usual dose $(0.1 \mathrm{~m} / 10 \mathrm{~kg})$ prior to the tumor resection to provide neuronavigation and after the resection to show the extent of resection and verify complete tumor resection. In case of poor visibility of the contrast-enhancing tumor, however, higher doses were reported to be applied at the discretion of the surgeon. However, there were notable differences between our patients with subarachnoid GBCA accumulation and those without.
ICU $1.25 d$

ICU $14.3 \pm 14 d$
According to the surgical reports, the tumor was frequently (5 of 7 patients) highly vascularized in the group of patients with subarachnoid GBCA accumulation, which led to excessive intraoperative bleeding and time-consuming hemostasis. Furthermore, in 1 patient a reresection had to be performed after the detection of residual tumor on the intraoperative MRI and, consequently, shortly after intraoperative GBCA application. In the control group, excessive bleeding was reported in 4 of 13 patients, but no GBCA was administered during surgery because those patients did not undergo intraoperative MRI. In the 6 patients in the control group with intraoperative MRI, no excessive bleeding or reresection have been reported. Therefore, a possible explanation for the GBCA accumulation after intraoperative MRI is that intraoperative bleeding and reresection shortly after GBCA application may lead to extravasation in the subarachnoid space, because the injected GBCA is presumably still present in the patients' circulation.

Thus, our small, descriptive data analysis suggests that GBCA accumulation possibly contributes to a clinical impairment after neurosurgical tumor resection. Consequently, GBCA extravasation and accumulation can be considered as a very rare but possible complication of intraoperative GBCA application in patients with resection of a hypervascularized tumor or reresection after intraoperative MRI. 


\section{Limitations}

Our study has several limitations. This is a retrospective case series on a very limited number of patients based on neuroradiological imaging analysis and interpretation of clinical data. Therefore, this series is considered as a merely descriptive analysis, because a statistical analysis cannot be performed due to the small sample size. However, given that GBCA leakage in high doses is a very rare condition, it is difficult to collect a larger cohort. Patients have been selected retrospectively based on visible subarachnoid GBCA accumulation; however, no quantitative measurement of signal changes in the subarachnoid space has been performed. Therefore, patients with a small amount of GBCA in the subarachnoid space were possibly not included, which presumably occurs in many patients with intraoperative GBCA application, and the vast majority might be asymptomatic.

On the other hand, visible signal changes of the CSF due to GBCA leakage suggest a larger amount of leaked GBCA and therefore might define a patient group at higher risk for neurotoxic complications. Furthermore, all patients received their postsurgical 3-T MRI within 12 to 72 hours, but not at the same time point after surgery. This might result in different grades of CSF signal changes. We tried to match the control group as closely as possible. However, although tumor characteristics and presurgical KPS scores did not differ significantly between both groups, not all patients in our control cohort underwent intraoperative MRI. However, the control subgroup of patients with intraoperative MRI did not significantly differ from the subgroup without it, with a trend toward better outcome and longer survival underlining the positive effect of intraoperative MRI.

\section{Conclusions}

Intraoperative MRI is known to be able to detect residual tumor-allowing immediate further tumor resection and being associated with extended PFS and OS. However, our small series suggests a possible risk of complications due to neurotoxic effects of intraoperatively applied GBCA if large doses leak into the subarachnoid space in cases of reresection or bleeding. The documentation of the GBCA dose and the awareness of the potential risk of complicating GBCA leakage in case of reresection or extensive bleeding might help avoid overdoses during intraoperative MRI. Besides deliberate overdosing, the accumulation is facilitated by impaired renal function and a short interval between pre- and intraoperative GBCA application. That is why it seems reasonable to perform the preoperative MRI 24 hours before surgery, to allow Gd to wash out, especially in patients with impaired renal function.

\section{References}

1. Senft C, Bink A, Franz K, et al. Intraoperative MRI guidance and extent of resection in glioma surgery: a randomised, controlled trial. Lancet Oncol. 2011;12(11):997-1003.

2. Brown TJ, Brennan MC, Li M, et al. Association of the extent of resection with survival in glioblastoma: a systematic review and meta-analysis. JAMA Oncol. 2016;2(11):1460-1469.

3. Messori A, Polonara G, Regnicolo L, et al. Effects of ionic and non-ionic paramagnetic contrast media on brain bioelectric activity. Neuroradiology. 2005;47(11):820-825.

4. Lescher S, Jurcoane A, Schniewindt S, et al. Misleading FLAIR imaging pattern after glioma surgery with intraoperative MRI. Neurosurg Rev. 2016;39(1):79-86.

5. Ray DE, Cavanagh JB, Nolan CC, Williams SC. Neurotoxic effects of gadopentetate dimeglumine: behavioral disturbance and morphology after intracerebroventricular injection in rats. AJNR Am J Neuroradiol. 1996;17(2):365-373.

6. Arlt S, Cepek L, Rustenbeck HH, et al. Gadolinium encephalopathy due to accidental intrathecal administration of gadopentetate dimeglumine. J Neurol. 2007;254(6):810-812.

7. Kapoor R, Liu J, Devasenapathy A, Gordin V. Gadolinium encephalopathy after intrathecal gadolinium injection. Pain Physician. 2010;13(5):E321-E326.

8. Stupp R, Mason WP, van den Bent MJ, et al. Radiotherapy plus concomitant and adjuvant temozolomide for glioblastoma. N Engl J Med. 2005;352(10):987-996.

9. Herrlinger U, Schäfer N, Steinbach JP, et al. Bevacizumab plus irinotecan versus temozolomide in newly diagnosed O6-methylguanine-DNA methyltransferase nonmethylated glioblastoma: the randomized GLARIUS trial. J Clin Oncol. 2016;34(14):1611-1619.

10. Tali ET, Ercan N, Krumina G, et al. Intrathecal gadolinium (gadopentetate dimeglumine) enhanced magnetic resonance myelography and cisternography: results of a multicenter study. Invest Radiol. 2002;37(3):152-159.

11. Zeng Q, Xiong L, Jinkins JR, et al. Intrathecal gadolinium-enhanced MR myelography and cisternography: a pilot study in human patients. AJR Am J Roentgenol. 1999;173(4):1109-1115.

12. Olchowy C, Cebulski K, Łasecki M, et al. The presence of the gadolinium-based contrast agent depositions in the brain and symptoms of gadolinium neurotoxicity-a systematic review. PLoS One. 2017;12(2):e0171704.

13. Sanai N, Polley MY, McDermott MW, et al. An extent of resection threshold for newly diagnosed glioblastomas. $J$ Neurosurg. 2011;115(1):3-8.

14. Mamourian AC, Hoopes PJ, Lewis LD. Visualization of intravenously administered contrast material in the CSF on fluid-attenuated inversion-recovery MR images: an in vitro and animal-model investigation. AJNR Am J Neuroradiol. 2000;21(1):105-111.

\section{Disclosures}

The authors report no conflict of interest concerning the materials or methods used in this study or the findings specified in this paper.

\section{Author Contributions}

Conception and design: Wagner, M Lauer. Acquisition of data: Wagner, M Lauer, Voss. Analysis and interpretation of data: Wagner, M Lauer, Voss. Drafting the article: Wagner, M Lauer. Critically revising the article: Wagner, A Lauer, You, Kluge, Hattingen, Harter, Senft, Voss. Reviewed submitted version of manuscript: Wagner. Approved the final version of the manuscript on behalf of all authors: Wagner. Study supervision: Wagner.

\section{Supplemental Information \\ Previous Presentations}

Portions of this work were presented in abstract form at the 2018 Annual Meeting of the German Society of Neuroradiology (DGNR), held in Frankfurt am Main, Germany, on October 3, 2018.

\section{Correspondence}

Marlies Wagner: Institute of Neuroradiology, University Hospital Frankfurt, Goethe University, Frankfurt am Main, Germany. marlieswa@gmx.de. 